\title{
Cost-effectiveness, cost-utility and the budget impact of antidepressants versus preventive cognitive therapy with or without tapering of antidepressants
}

Nicola S. Klein, Ben F. M. Wijnen, Joran Lokkerbol, Erik Buskens, Hermien J. Elgersma, Gerard D. van Rijsbergen, Christien Slofstra, Johan Ormel, Jack Dekker, Peter J. de Jong, Willem A. Nolen, Aart H. Schene, Steven D. Hollon, Huibert Burger and Claudi L. H. Bockting

\section{Background}

As depression has a recurrent course, relapse and recurrence prevention is essential.

\section{Aims}

In our randomised controlled trial (registered with the Nederlands trial register, identifier: NTR1907), we found that adding preventive cognitive therapy (PCT) to maintenance antidepressants (PCT+AD) yielded substantial protective effects versus antidepressants only in individuals with recurrent depression. Antidepressants were not superior to PCT while tapering antidepressants (PCT/-AD). To inform decision-makers on treatment allocation, we present the corresponding costeffectiveness, cost-utility and budget impact.

\section{Method}

Data were analysed $(n=289)$ using a societal perspective with 24-months of follow-up, with depression-free days and qualityadjusted life years (QALYS) as health outcomes. Incremental cost-effectiveness ratios were calculated and cost-effectiveness planes and cost-effectiveness acceptability curves were derived to provide information about cost-effectiveness. The budget impact was examined with a health economic simulation model.

\section{Results}

Mean total costs over 24 months were $€ 6814, € 10264$ and $€ 13282$ for AD+PCT, antidepressants only and PCT/-AD, respectively. Compared with antidepressants only, $P C T+A D$ resulted in significant improvements in depression-free days but not QALYS. Health gains did not significantly favour antidepressants only versus PCT/-AD. High probabilities were found that $\mathrm{PCT}+\mathrm{AD}$ versus antidepressants only and antidepressants only versus PCT/-AD were dominant with low willingness-to-pay thresholds. The budget impact analysis showed decreased societal costs for PCT+AD versus antidepressants only and for antidepressants only versus PCT/-AD.

\section{Conclusions}

Adding PCT to antidepressants is cost-effective over 24 months and PCT with guided tapering of antidepressants in long-term users might result in extra costs. Future studies examining costs and effects of antidepressants versus psychological interventions over a longer period may identify a break-even point where PCT/-AD will become cost-effective.

\section{Declaration of interest}

C.L.H.B. is co-editor of PLOS One and receives no honorarium for this role. She is also co-developer of the Dutch multidisciplinary clinical guideline for anxiety and depression, for which she receives no remuneration. She is a member of the scientific advisory board of the National Insure Institute, for which she receives an honorarium, although this role has no direct relation to this study. C.L.H.B. has presented keynote addresses at conferences, such as the European Psychiatry Association and the European Conference Association, for which she sometimes receives an honorarium. She has presented clinical training workshops, some including a fee. She receives royalties from her books and co-edited books and she developed preventive cognitive therapy on the basis of the cognitive model of A. T. Beck. W.A.N. has received grants from the Netherlands Organisation for Health Research and Development and the European Union and honoraria and speakers' fees from Lundbeck and Aristo Pharma, and has served as a consultant for Daleco Pharma.

\section{Keywords}

Depressive disorders; antidepressants; cognitive behavioural therapies; cost-effectiveness; economics.

\section{Copyright and usage}

(C) The Royal College of Psychiatrists 2019. This is an Open Access article, distributed under the terms of the Creative Commons Attribution-NonCommercial-NoDerivatives licence (http://creativecommons.org/licenses/by-nc-nd/4.0/), which permits noncommercial re-use, distribution, and reproduction in any medium, provided the original work is unaltered and is properly cited. The written permission of Cambridge University Press must be obtained for commercial re-use or in order to create a derivative work.
Major depressive disorder (MDD) is a highly prevalent and disabling disorder ${ }^{1}$ characterised by a recurrent nature. ${ }^{2}$ Besides the substantial disability burden, MDD poses considerable financial consequences because of healthcare use and productivity losses. ${ }^{3}$ To reduce the disability and economic burden of MDD, it is important to focus on strategies that are effective in preventing relapse/recurrence (further referred to as recurrence). ${ }^{4}$ Antidepressants are a widely used relapse prevention strategy and protect against recurrence, with odds ratios ranging between 0.30 and 0.48 compared with discontinuing antidepressants and switching to placebo (for meta-analyses, see
Borges et al, Geddes et al, Glue et al and Kaymaz et $\left.a l^{5-8}\right)$. However, non-adherence is common ${ }^{9}$ and $75 \%$ of individuals favour psychological interventions over antidepressants. ${ }^{10}$ In past decades, the effectiveness of psychological relapse prevention strategies for MDD has been substantiated. ${ }^{4}$ However, economic research has not kept pace with the development of relapse prevention strategies. The increased emphasis on evidence-based psychiatry coincides with a need to examine and document the wider societal costs and benefits of treatments to inform decisions regarding allocation and reimbursement of treatments in mental healthcare. 
In the Disrupt the Rhythm of Depression (DRD) trial (registered with the Nederlands trial register, identifier: NTR1907), ${ }^{11}$ we found that adding preventive cognitive therapy (PCT) to maintenance antidepressants $(\mathrm{PCT}+\mathrm{AD})$ resulted in a statistically significant risk reduction in terms of time-related proportion of individuals with depressive recurrence compared with maintenance antidepressants only. We could not demonstrate that antidepressants reduced the risk of recurrence more compared with PCT with guided tapering of antidepressants (PCT/-AD). ${ }^{12}$ The aim of the current study was to perform cost-effectiveness, cost-utility and budget impact analyses alongside the DRD trial.

\section{Method}

\section{Study design}

The DRD trial is a single-blind multicentre three-arm randomised controlled trial. ${ }^{11}$ The economic evaluation was performed according to the Dutch guidelines ${ }^{13}$ and reported according to the Consolidated Health Economic Evaluation Reporting Standards statement. ${ }^{14}$ The study was approved by an independent medical ethics committee (METIGG) and described in detail elsewhere. ${ }^{11,12}$

\section{Participants and procedure}

Participants were recruited via general practitioners, pharmacists, secondary mental healthcare and the media. To be included, participants had to have experienced at least two prior major depressive episodes, had been in remission for at least 8 weeks but no longer than 2 years based on DSM-IV criteria as assessed by the Structured Clinical Interview for DSM-IV Axis-I Disorders $(\text { SCID-I })^{15}$ and have a score on the Hamilton Rating Scale for Depression $^{16}$ of $\leq 10$. For the full definition of relapse/recurrence and remission/recovery see supplementary File 1 available at https://doi.org/10.1192/bjp.2018.81. Participants must have used maintenance antidepressants for at least 6 months. Exclusion criteria were (hypo)mania or a psychotic disorder/MDD with psychotic features, current alcohol or drug misuse, organic brain damage, a predominant anxiety disorder or psychotherapy more than twice a month. Because individuals with various depressive episodes over a longer time span also benefit from relapse prevention strategies, ${ }^{4}$ we discarded our initial criterion that both major depressive episodes must have occurred within the past 5 years. In addition, PCT was initially offered in groups but we extended this to individual sessions during the trial since many participants were not able to attend the group meetings because of practical concerns.

\section{Randomisation}

After obtaining informed consent, participants were randomised to $\mathrm{PCT}+\mathrm{AD}$, antidepressants only or PCT/-AD by an independent research assistant not otherwise involved in the study using automated stratified permuted block randomisation with computergenerated random numbers (allocation ratio 10:10:8, respectively). The randomisation was stratified by number of previous major depressive episodes (two versus three or more) and baseline care (general practitioner versus secondary mental healthcare). Participants were informed about their treatment allocation by a research assistant not involved in the follow-up interviews. Independent assessors masked to treatment allocation conducted the follow-up interviews.

\section{Interventions}

PCT is a treatment strategy that is effective in preventing recurrence in individuals in remission with recurrent depression ${ }^{17-21}$ and targets potential cognitive vulnerability factors of MDD. It consists of eight manualised individual or group sessions performed by trained psychologists. Issues with therapist adherence were discussed and resolved during supervision meetings. An independent research assistant assessed therapist adherence to the treatment manual and adherence was judged to be high (87\%, range: $81-95$, for details, see Bockting et $a l^{12}$ ). In the two antidepressant continuation groups, general practitioners and psychiatrists were advised to continue prescribing antidepressants at the minimal required adequate dosage or higher ( $\geq 20 \mathrm{mg}$ fluoxetine equivalent) and in the tapering group to taper the participants' antidepressant within 4 weeks. Participants' adherence to the randomised condition is described in detail elsewhere. ${ }^{12}$ In short, adherence to PCT (i.e. completing at least five sessions) was $88 \%$ in the $\mathrm{PCT}+\mathrm{AD}$ group and $90 \%$ in the $\mathrm{PCT} /-\mathrm{AD}$ group. Adherence to antidepressants was monitored with the Trimbos and iMTA questionnaire on costs associated with psychiatric illness (TiC-P). ${ }^{22}$ Most (60\%) individuals in the $\mathrm{PCT} /-\mathrm{AD}$ group tapered their ADs over 6 months. At 6 months, adherence to antidepressants was $58 \%$ in the antidepressants-only group, $60 \%$ in $\mathrm{PCT} /-\mathrm{AD}$ group and $65 \%$ in the $\mathrm{PCT}+\mathrm{AD}$ group.

\section{Economic evaluation}

Costs

In accordance with the Dutch guidelines, we used a societal perspective in this economic evaluation, in which all costs inside and outside the healthcare sector were assessed. We used the TiC-P, ${ }^{22}$ which is a reliable and valid self-report instrument for collecting cost data, ${ }^{23}$ to prospectively assess the costs. The TiC-P was designed to refer to the past 3 months and was administered at baseline and subsequently at 3-month intervals, with the exception of the last assessment which had a 9-month interval. For the costs in this 9-month interval we therefore took the costs of the past 3 months multiplied by three. In addition, the maximum number of days medicated was only 28 and therefore we extrapolated all medication use to 3 months.

Costs within the healthcare sector were related to a range of healthcare services participants used during the study, including medication and in-patient stays, and out-patient and primary care appointments. Costs related to PCT included training and supervision of therapists, costs of contacts between participants and therapists and costs of the workbooks used by participants. Patient and family costs included informal care (i.e. the monetary valuation of time invested by relatives or acquaintances in assisting the participant), travel expenses associated with healthcare visits and (psychiatric) home care. Informal care was measured as part of the TiC-P, by asking participants to express the number of hours per week they received care from relatives and/or friends. This was then valued using the proxy good method (i.e. time spent on caregiving was valued at the (labour) market price of a close substitute, in this case housekeeping). Productivity losses were quantified and included absence from work (absenteeism), reduced productivity while at work (presenteeism) and productivity losses of unpaid work. We used the friction cost method to estimate costs associated with productivity losses as a result of illness-related absence from work. ${ }^{24}$ To promote comparisons with other economic evaluations, Dutch standard prices ${ }^{25}$ were used as unit prices. We estimated true costs of used resources when standard prices were not available. All unit prices were based on the price level of the Euro in 2015. Reference prices established for previous years were adjusted to 2015 prices applying the consumer price index. 


\section{Outcomes}

The health outcome of the cost-effectiveness analysis was the number of depression-free days over a period of 24 months based on DSM-IV criteria assessed with the SCID-I by masked interviewers after 3, 9, 15 and 24 months. Quality-adjusted life-years (QALYs) over 24 months was the health outcome measure in the cost-utility analysis and represent disease burden by combining quality (expressed in utilities) and quantity of life, where one QALY represents 1 year of perfect health. We used utilities, derived from the EQ-5D-3L that measures health-related quality of life, ${ }^{26}$ to calculate QALYs with the area under the curve method. These utilities were calculated with Dutch tariffs to obtain utilities for specific health states. ${ }^{27}$ Costs and health outcomes were discounted in accordance with current Dutch guidelines (1.5\% health outcomes, $4 \%$ costs). ${ }^{25}$

\section{Statistical analyses}

This study was conducted alongside a clinical trial and the power calculation of the primary outcome is provided elsewhere. ${ }^{11}$ This power calculation was based on detecting a difference in the timerelated proportion of individuals with depressive recurrence regarding the following comparisons: (a) adding PCT to antidepressants versus antidepressants only; and (b) antidepressants only versus PCT while tapering antidepressants. Given that the study was only powered to detect a difference in a depression-related outcome and not in costs, we used probabilistic and medical decision-making techniques to draw inferences about cost-effectiveness. Conforming to the trial protocol, the analyses in this manuscript were restricted to examine these two comparisons with as primary analysis a cost-effectiveness analysis using depression-free days as the health outcome and as the secondary analysis a cost-utility analysis using QALYs as the health outcome.

We used the intention-to-treat principle, in which all participants were included in the analyses regardless of adherence to the randomised interventions. To deal with missing data, multiple imputations by chained equations with predictive mean matching, which is recommended for dealing with missing data in (cost-effectiveness) trials ${ }^{28,29}$ were used in our main analysis, incorporating baseline variables predictive of outcome and drop-out. Multiple imputations were used to make optimal use of the available data and reduce possible selection bias because of non-random dropout. Costs and outcomes associated with each treatment condition were used to calculate the incremental cost-effectiveness ratio (ICER) relative to an alternative. ${ }^{30}$ The formula of the ICER is:

$$
\mathrm{ICER}=\frac{\left(\mathrm{C}_{\mathrm{PCT}+\mathrm{AD}}-\mathrm{C}_{\mathrm{AD}}\right)}{\left(\mathrm{QALY} \mathrm{PCT}_{\mathrm{P}} \mathrm{AD}-\mathrm{QALY} \mathrm{AD}_{\mathrm{AD}}\right)}
$$

where $\mathrm{C}_{\mathrm{PCT}+\mathrm{AD}}$ is the mean costs in the $\mathrm{PCT}+\mathrm{AD}$ group; $\mathrm{C}_{\mathrm{AD}}$ is the mean costs in the antidepressants-only group; $\mathrm{QALY}_{\mathrm{PCT}+\mathrm{AD}}$ is the mean QALYS in the PCT+AD group and $\mathrm{QALY}_{\mathrm{AD}}$ is the mean QALYS in the antidepressants-only group.

The bootstrap method ${ }^{31}$ was used for information about the uncertainty of the results. To allow correlated residuals and correct for differences in baseline characteristics in sensitivity analyses, seemingly unrelated regression equations were bootstrapped 5000 times. Simulated values of the estimates for cost and outcome differences were displayed in a cost-effectiveness plane ${ }^{32}$ to capture the uncertainty in the ICER estimate. Information about the cost-effectiveness plane can be found in supplementary File 2. Cost-effectiveness acceptability curves (CEACs) ${ }^{33}$ were derived that inform decision-makers about the probability that an intervention will be cost-effective, depending on the willingness to pay per additional unit of health outcome.
Both complete cost and effect data were available for 105 participants. For 169 participants at least $50 \%$ of the cost data and for 175 at least $50 \%$ of the QALY data were available during the 24 months. For 197 participants follow-up data on depression-free days were available. We performed several sensitivity analyses to examine the robustness of our results. The intervention could not be started and follow-up data were not available for 43 participants in the PCT groups for practical reasons. In addition, 16 participants in the PCT groups and 21 in the antidepressants-only group dropped out immediately after randomisation for other reasons. Ultimately, 209 participants had follow-up data and were used in our main analysis. We included all 289 participants in a sensitivity analysis.

Furthermore, we repeated the analyses in participants with at least $50 \%$ cost data available and in complete cases. We also repeated the main analysis while correcting for baseline costs and utilities, for whether participants filled out additional momentary assessments, and for whether participants in the therapy groups received the individual or group therapy. Finally, our results for the primary outcomes suggested an increased recurrence rate for PCT/-AD compared with antidepressants only in the first 140 days. ${ }^{12}$ To account for possible withdrawal symptoms in the first months, we performed an analysis taking into account only the final 1.5 years of the study.

\section{Budget impact analysis}

We used a health economic simulation model for depression, DEPMOD, ${ }^{34}$ to examine the budget impact when offering the different relapse prevention strategies to an estimated $25 \%$ of the target population in the Netherlands. Per-person cost differences as estimated by the trial data were applied to $25 \%$ of the target group to estimate the order of magnitude of the budget impact. The budget impact was estimated both from a healthcare perspective and a societal perspective, and considered a 2-year time horizon.

\section{Results}

Details of the participant flow have been described elsewhere ${ }^{12}$ Between 14 July 2009 and 30 April 2015, 289 participants were assessed for eligibility and randomised to the PCT+AD $(n=104)$, antidepressants-only $(n=100)$ or PCT/-AD $(n=85)$ groups. Of those, 209 provided additional data following randomisation. Demographic and clinical variables are displayed in Table 1 . The participants in the treatment groups appeared to have similar characteristics, except for slight imbalances in marital status, education and employment status. Since baseline characteristics of all participants and the participants with any follow-up data were similar and equally distributed over the treatment groups, no indication for a systematic bias because of drop-out was found.

\section{Costs}

The various types of costs generated by the three groups and information on the use of healthcare services during the 24 months of the study are presented in supplementary Table 1 . Costs are based on the data of participants for whom at least one cost measurement was available during follow-up. Mean costs per participant directly related to PCT were $€ 349, € 354$, and $€ 0$ in the PCT+AD, PCT/-AD and antidepressants-only groups, respectively. These costs mainly consisted of costs related to training/supervision of therapists and contacts between participants and therapists. Hospital admissions and care provided by mental healthcare institutions contributed considerably to overall costs within the healthcare sector. Costs associated with productivity losses were substantial. 


\begin{tabular}{|c|c|c|c|}
\hline & PCT+AD group $(n=104)$ & Antidepressants-only group $(n=100)$ & PCT/-AD group $(n=85)$ \\
\hline Age, years: ${ }^{a}$ mean (s.d.) & $47.0(9.3)$ & $47.2(10.5)$ & $47.7(11.1)$ \\
\hline Gender, women: $n(\%)$ & $72(69)$ & $64(64)$ & $53(62)$ \\
\hline Dutch nationality, $n$ (\%) & $101(97)$ & 95/99 (96) & $82 / 84(98)$ \\
\hline \multicolumn{4}{|l|}{ Marital status, ${ }^{,} n(\%)$} \\
\hline Single & $27 / 103(26)$ & 32/99 (32) & 28/84 (33) \\
\hline Married/cohabiting & 69/103 (67) & $59 / 99(60)$ & $46 / 84(55)$ \\
\hline Divorced/widowed & 7/103 (7) & 8/99 (8) & 10/84 (12) \\
\hline \multicolumn{4}{|l|}{ Education, ${ }^{\mathrm{b}} \mathrm{n}(\%)$} \\
\hline Primary and/or secondary education & 20 (19) & 25/99 (25) & 12/84 (14) \\
\hline Vocational education & $31(30)$ & 28/99 (28) & 23/84 (27) \\
\hline Higher education & $53(51)$ & 46/99 (46) & 49/84 (58) \\
\hline Employed, ${ }^{\mathrm{b}} n(\%)$ & 73/103 (71) & $65 / 98(66)$ & $53 / 84(63)$ \\
\hline \multicolumn{4}{|l|}{ Treatment as usual, $n$ (\%) } \\
\hline Specialised mental healthcare & $32(31)$ & $31(31)$ & $26(31)$ \\
\hline General practitioner & $72(69)$ & $69(69)$ & $59(69)$ \\
\hline Number of depressive episodes, median (IQR) & $5(3-6)$ & $4(3-6)$ & $5(3-6)$ \\
\hline Total HRSD, mean (s.d.) & $3.6(3.1)$ & $3.8(3.1)$ & $3.6(3.0)$ \\
\hline Total IDS-SR, mean (s.d.) & $20.4(11.5)$ & $18.5(10.8)$ & $20.6(12.1)$ \\
\hline \multicolumn{4}{|l|}{ Type of antidepressant, ${ }^{b} n(\%)$} \\
\hline SSRI & 85/103 (83) & 79/99 (80) & $69 / 85(81)$ \\
\hline SNRI & 1/103 (1) & 8/99 (8) & $1 / 85(1)$ \\
\hline Tricyclic antidepressant & 7/103 (7) & 7/99 (7) & 10/85 (12) \\
\hline Atypical antidepressant & $5 / 103(5)$ & 2/99 (2) & $3 / 85$ (4) \\
\hline Monoamine oxidase inhibitor & $0(0)$ & 1/99 (1) & $0(0)$ \\
\hline More than one antidepressant & $5 / 103(5)$ & 2/99 (2) & 2/85 (2) \\
\hline EQ-5D-3L, ${ }^{C}$ mean (s.d.) & $0.84(0.16)$ & $0.80(0.18)$ & $0.79(0.17)$ \\
\hline Baseline costs, ${ }^{c} €$ : mean (s.d.) & $1533(5423)$ & 1695 (3049) & 1778 (3383) \\
\hline
\end{tabular}

When visually inspecting supplementary Table 1 , in most categories costs appear slightly lower for the $\mathrm{PCT}+\mathrm{AD}$ group compared with the antidepressants-only group and the antidepressantsonly group compared with the $\mathrm{PCT} /-\mathrm{AD}$ group except for larger reductions for $\mathrm{PCT}+\mathrm{AD}$ compared to antidepressants-only regarding absenteeism and hospital admissions.

An overview of the mean costs per measurement for all 209 individuals is displayed in supplementary Table 2. Mean total costs of the PCT+AD group appear lower than in the two other groups at each measurement period, except for the first measurement (0-3 months). Mean total costs for the antidepressants-only group compared with the $\mathrm{PCT} / \mathrm{AD}$ group appear only higher between 3 and 9 months and lower during the other measurements. Accumulating all costs (supplementary Table 2), mean total costs during the 24 months of the study were $€ 6814$ for the PCT+AD group, $€ 10264$ for the antidepressants-only group and $€ 13282$ for the $\mathrm{PCT} / \mathrm{AD}$ group.

\section{Effects}

The mean number of depression-free days within 24 months followup was 628 (range 187-730) for the antidepressants-only group, 607 (range 51-730) for the PCT/-AD group and 662 (range 194-730) for the $\mathrm{PCT}+\mathrm{AD}$ group. A statistically significant difference in depression-free days was found for the $\mathrm{PCT}+\mathrm{AD}$ group compared with the antidepressants-only group $(P=0.016)$. The difference in depression-free days for the antidepressants-only group compared with the PCT/-AD group was not statistically significant $(P=$ 0.637). Mean QALYs over 24 months were 1.62 (range 0.95-1.95) for the PCT+AD group, 1.64 (range 1.00-1.99) for the antidepressants-only group and 1.59 (range $0.64-1.94$ ) for the PCT/-AD group. No statistically significant differences in QALYs were found between the PCT $+\mathrm{AD}$ group compared with the antidepressants-only group $(P=0.907)$ and the antidepressantsonly group compared with the $\mathrm{PCT} /-\mathrm{AD}$ group $(P=0.628)$.

\section{Economic evaluation}

The results of the main analyses are presented in Table 2. Table 2 and the cost-effectiveness plane (Fig. 1) show that regarding depression-free days, most $(93.1 \%)$ of the bootstrapped ICERs were located in the south-east quadrant, indicating a $93.1 \%$ probability that costs were lower and health outcomes better for PCT+AD compared with the antidepressants-only group (i.e. PCT $+\mathrm{AD}$ is dominant). The CEAC in Fig. 1 regarding depression-free days shows a high probability that PCT $+\mathrm{AD}$ is dominant. Regarding QALYs, $\mathrm{PCT}+\mathrm{AD}$ was associated with $67.8 \%$ of the bootstrapped ICERs appearing in the south-west quadrant, indicating that costs were lower and health outcomes worse compared with antidepressants only (Table 2 and Fig. 1). Interpretation of outcomes located in the south-west quadrant depends on whether decision-makers are willing to accept a cost reduction for a loss in health. The CEAC in Fig. 1 regarding QALYs shows a high probability that $\mathrm{PCT}+\mathrm{AD}$ dominates antidepressants only when the willingnessto-accept threshold is low and a decreasing probability if the threshold to accept a reduction in health is increased.

Regarding antidepressants only compared with PCT/-AD, Table 2 and the cost-effectiveness planes (Fig. 2) show that most (72.9\% for depression-free days and $80.8 \%$ for QALYs) of the bootstrapped ICERs were located in the south-east quadrant, indicating a $72.9 \%$ and $80.8 \%$ probability that antidepressants only generated lower costs and better outcomes compared with PCT/-AD (i.e. antidepressants only is dominant). The CEACs in Fig. 2 show that the probability that antidepressants only are dominant compared with $\mathrm{PCT} / \mathrm{AD}$ is approximately $90 \%$ for both depression-free days and QALYs when the willingness to pay per additional 


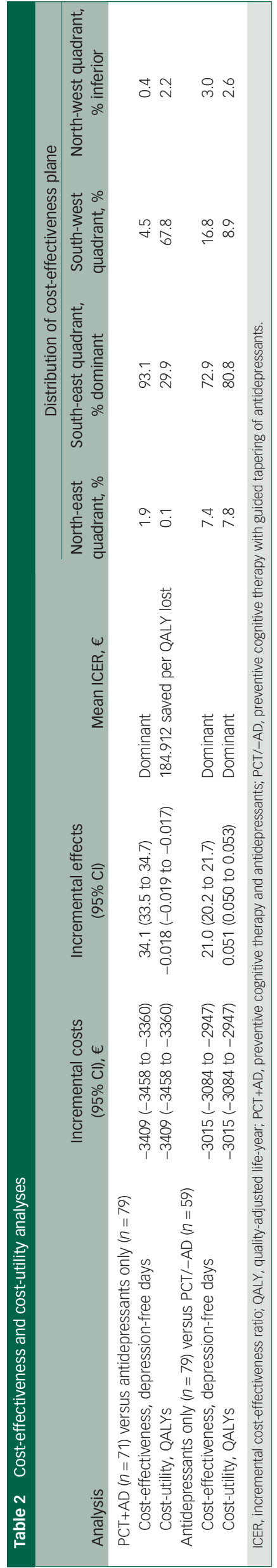

health gain is zero and the probability for depression-free days slightly decreases and for QALYs slightly increases with additional investments. The sensitivity analyses yielded similar results. When only taking into account the last 1.5 years of the study to examine the cost-effectiveness of antidepressants only compared with $\mathrm{PCT} / \mathrm{AD}$, the gain in depression-free days decreased from 21 to 12 but antidepressants only remained cost-effective compared with $\mathrm{PCT} / \mathrm{-AD}$.

\section{Budget impact analysis}

The target population size in the Netherlands in a given year, meaning the yearly prevalence of people with at least two previous episodes of depression, was estimated to be 110000 , which is roughly $1 \%$ of the total population of approximately 10 million people aged between 18 and 65 in the Netherlands. Offering PCT $+A D$ instead of antidepressants alone to $25 \%$ of the target population is associated with an estimated decrease in costs of 76 million euro from a healthcare perspective and 95 million euro from a societal perspective. Offering antidepressants alone instead of $\mathrm{PCT} / \mathrm{AD}$ to $25 \%$ of the target population is associated with an estimated decrease in costs of 9.5 million euro from a healthcare perspective and 83 million euro from a societal perspective.

\section{Discussion}

Adding PCT to antidepressants had the highest number of depression-free days and lowest costs, PCT/-AD had the lowest number of depression-free days and highest costs, and antidepressants only ranked in-between in terms of depression-free days and costs. Adding PCT to antidepressants was dominant compared with antidepressants only regarding depression-free days and resulted in decreased costs at a population level whereas antidepressants only dominated $\mathrm{PCT} / \mathrm{AD}$ in the cost-effectiveness, cost-utility and budget impact analyses.

\section{Adding PCT to antidepressants compared with antidepressants only}

Although adding PCT to antidepressants was dominant compared with antidepressants only resulting in an increase (statistically significant) in depression-free days and decrease in costs, QALYs did not significantly differ for PCT $+\mathrm{AD}$ compared with antidepressants only. The EQ-5D used in our study might lack sensitivity to detect small improvements in individuals in remission with recurrent MDD. Moreover, it only displays the current health state and therefore does not capture all recurrences during the 24 months of the study. Studies indeed suggest that the EQ-5D might be less representative for individuals with psychiatric symptoms. ${ }^{35}$ Therefore, also in line with the trial protocol, we consider the cost-effectiveness analysis using depression-free days as the primary outcome.

The finding that $\mathrm{PCT}+\mathrm{AD}$ dominated antidepressants only in terms of depression-free days contrasts with another Dutch relapse prevention study where adding mindfulness-based cognitive therapy to maintenance antidepressants did not reduce the risk of recurrence compared with antidepressants only. ${ }^{36}$ However, it is congruent with studies demonstrating that sequential cognitive therapy after remission is protective of recurrence ${ }^{37}$ and with our primary outcomes. ${ }^{12}$ Moreover, our finding is partly in line with a cost-effectiveness study of a relapse prevention strategy for partially remitted depression that found cognitive therapy added to antidepressants and clinical management was likely to be cost-effective compared with antidepressants and clinical management only over 17 months when decision-makers were willing to pay $£ 4500$ per recurrence prevented, ${ }^{38}$ and with a cost-effectiveness study 
(a)

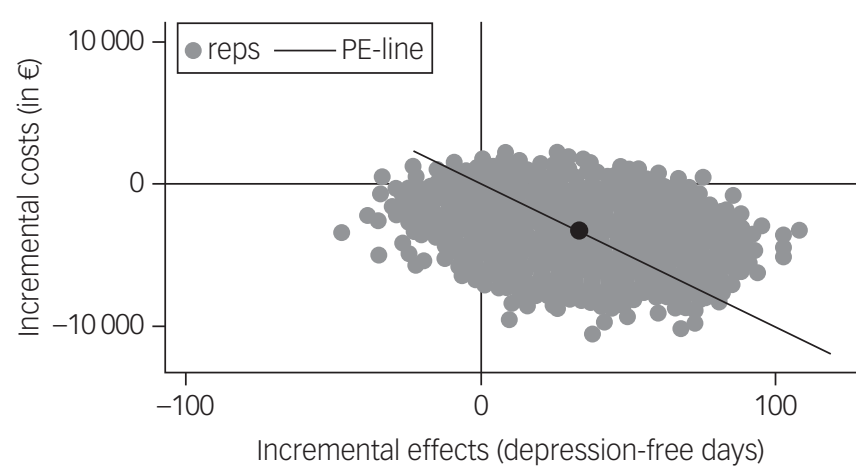

(c)

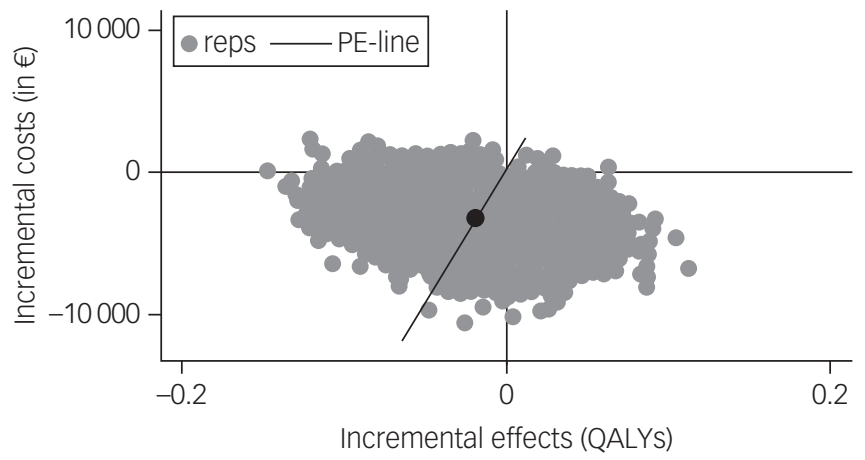

(b)

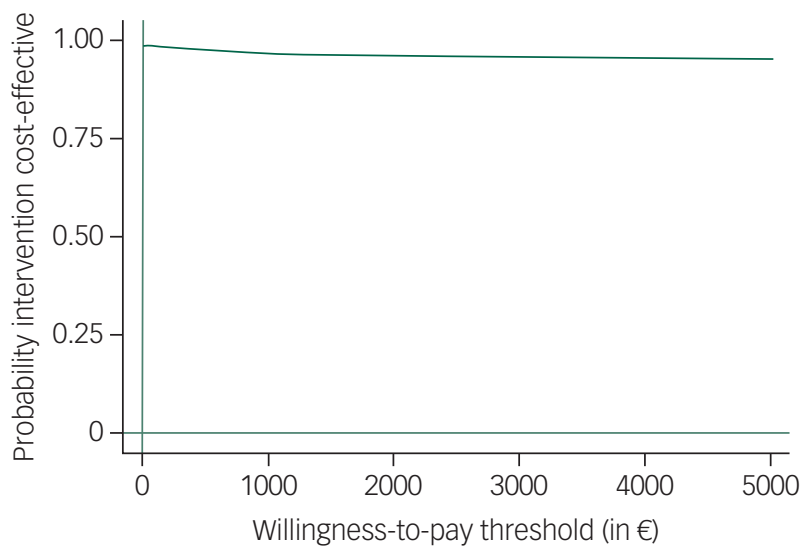

(d)

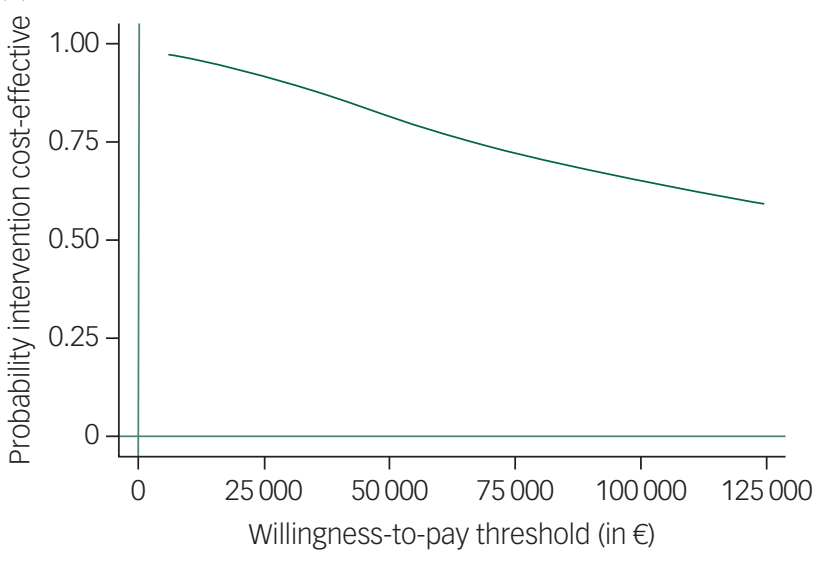

Fig. 1 Incremental cost-effectiveness planes ((a) and (c)) and cost-effectiveness acceptability curves ((b) and (d)) for costs per depression-free day ((a) and (b)) and costs per quality-adjusted life-years (QALYS) gained ((c) and (d)) for preventive cognitive therapy plus antidepressants compared with antidepressants only.

Based on 5000 bootstrapped cost-effectiveness pairs. Reps, bootstrap replication; PE-line, line that represents the point estimate of the incremental cost-effectiveness ratio (average cost/effect of bootstrap replications).

regarding a relapse prevention strategy for individuals in (partial) remission using maintenance antidepressants that found family psychoeducation added to treatment as usual was highly likely to be cost-effective compared with treatment as usual over 9 months if a depression-free day would be valued at $\$ 20$ or more. ${ }^{39}$ In both studies, only direct healthcare costs were taken into account.

Karyotaki et $a l^{40}$ performed a systematic review of economic evaluations alongside randomised controlled trials for depression treatments. Three trials were identified comparing the combination of a psychological intervention and antidepressants versus antidepressant only and inconsistent results were found. Overall, Karyotaki et $a l^{40}$ concluded that studies likely varied widely in results because of differences in study design and study population and that there remain important gaps in knowledge regarding economic evaluations for MDD treatments. Our study is the first to examine the economic consequences of adding a psychological intervention to maintenance antidepressants in individuals who are recurrently depressed and it shows promising results. More studies are needed to substantiate this finding.

\section{Antidepressants only compared with PCT while tapering antidepressants}

Health outcomes did not significantly favour antidepressants only compared with $\mathrm{PCT} /-\mathrm{AD}$, which is in line with our primary outcome. ${ }^{12}$ However, the cost-effectiveness plane showed that antidepressants only dominated PCT/-AD as most of the bootstrapped ICERs were located in the south-east quadrant where costs are lower and health outcomes better. Antidepressants only also resulted in lower societal costs in the budget impact analysis. These findings are only partly consistent with the findings of two studies examining the (cost)effectiveness of a relapse prevention strategy (mindfulness-based cognitive therapy) with support to taper off maintenance antidepressants compared with maintenance antidepressants only in individuals in remission from recurrent depression. ${ }^{41,42}$ In one study, antidepressants dominated mindfulness-based cognitive therapy with tapering support when the willingness-to-pay threshold was zero per additional recurrence prevented but this reversed when the willingness to pay increased to $\$ 1000$ and above. ${ }^{41}$ Results of the other study also suggested that an improvement in terms of recurrence was achieved at higher costs for mindfulnessbased cognitive therapy while tapering antidepressants compared with antidepressants only, but that antidepressants dominated mindfulness-based cognitive therapy while tapering antidepressants in terms of QALYs. The probability that mindfulness-based cognitive therapy was cost-effective compared with antidepressants did not rise above $52 \%$ regardless of effect measure. ${ }^{42}$

As also stated in the DRD study, ${ }^{12}$ it is important to examine why the results were not better for PCT/-AD compared with antidepressants only, whereas the addition of PCT to antidepressants 
(a)

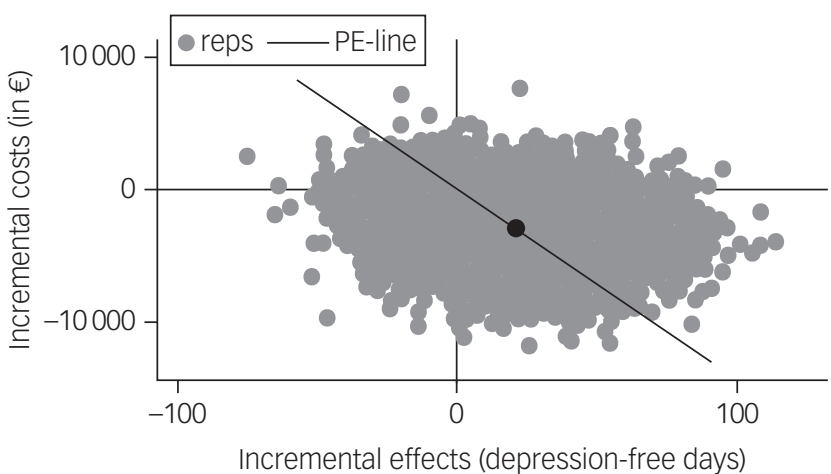

(c)

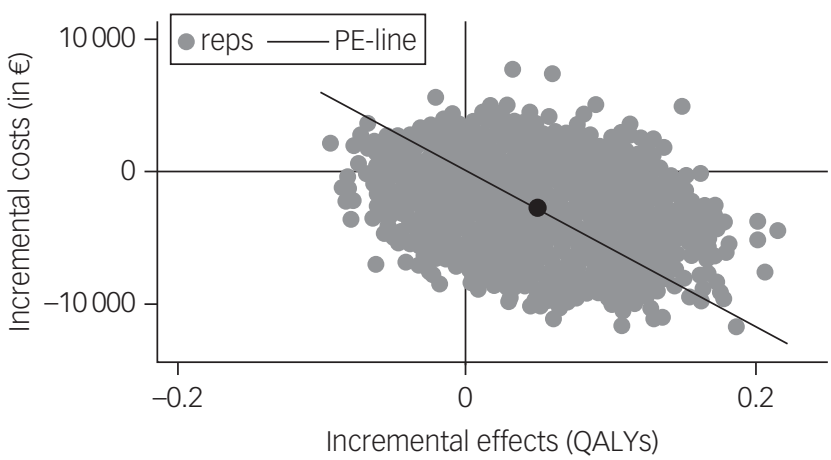

(b)

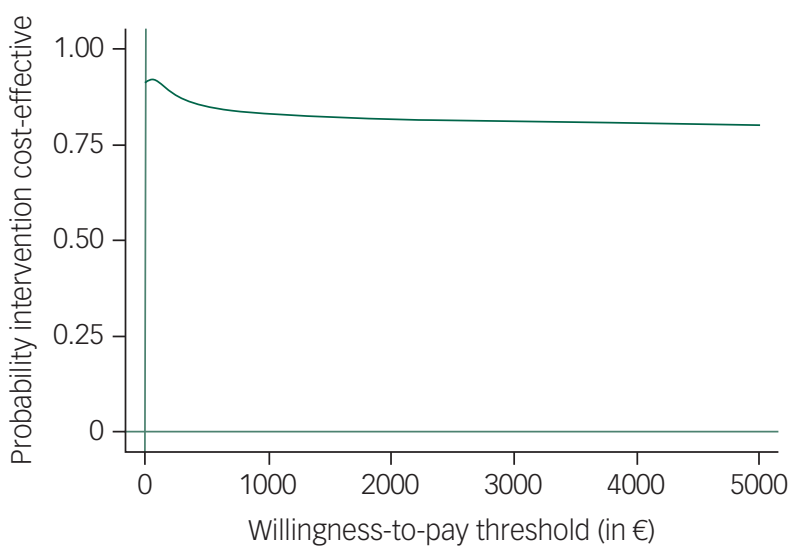

(d)

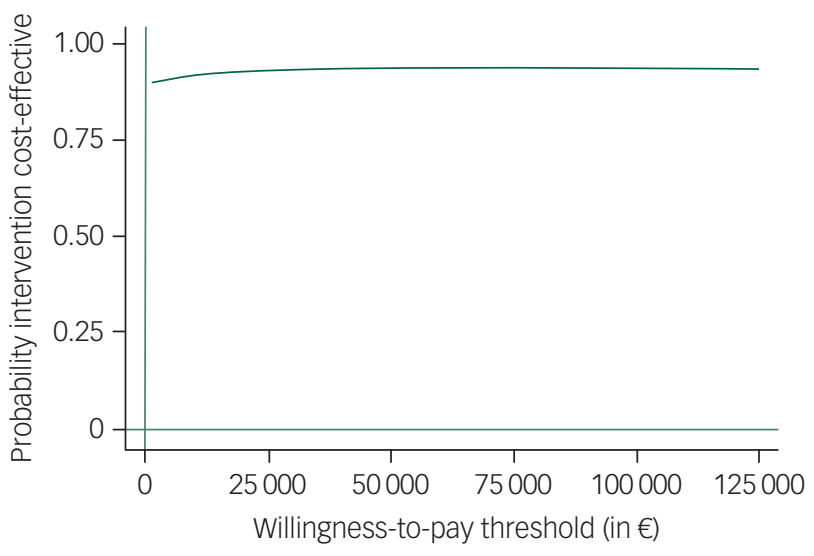

Fig. 2 Incremental cost-effectiveness planes ((a) and (c)) and cost-effectiveness acceptability curves ((b) and (d)) for costs per depression-free day ((a) and (b)) and costs per quality-adjusted life-years (QALYS) gained ((c) and (d)) for antidepressants only compared with preventive cognitive therapy with guided tapering of antidepressants.

Based on 5000 bootstrapped cost-effectiveness pairs. Reps, bootstrap replication; PE-line, line that represents the point estimate of the incremental cost-effectiveness ratio (average cost/effect of bootstrap replications).

resulted in additional protection. One explanation might be a temporary imbalance caused by withdrawal of antidepressants. The systematic review of Fava et al ${ }^{43}$ suggests that withdrawal symptoms can take many forms and that they usually occur within a few days or weeks but can also persist for a longer period (i.e. up to 1 year). This corroborates the DRD study, where we found a higher risk of recurrence during the first 140 days gradually disappearing thereafter in participants tapering antidepressants with PCT compared with antidepressant only. ${ }^{12}$ However, when correcting for this possible destabilisation in the current study, the gain in depression-free days for antidepressants only decreased but costs remained lower and effects slightly better for antidepressants only compared with $\mathrm{PCT} /-\mathrm{AD}$. It should be noted that most participants (60\%) tapered their antidepressants within 6 months, indicating that the advised 4 weeks is not feasible for many and that possible withdrawal effects may have occurred later during the study.

A second explanation might be that participants had difficulty tapering off antidepressants, resulting in a cycling on and off antidepressants that has shown to generate high $\operatorname{costs}^{44}$ and might result in progressive tolerance to antidepressants. ${ }^{45} \mathrm{~A}$ third explanation might be that prolonged use of antidepressants results in oppositional tolerance, heightening the recurrence risk after discontinuation. ${ }^{46}$

In the long term, we expect costs and outcomes to be more promising for $\mathrm{PCT} / \mathrm{AD}$ compared with antidepressants only as costs of antidepressants will be reduced, and outcomes will be enhanced because of the enduring effects of PCT up to 10 years. ${ }^{4,18}$ This is also in line with two cost-effective modelling studies in episodic and maintenance MDD that showed maintenance cognitive-behavioural therapy and antidepressants were both cost-effective strategies over 5 years, with maintenance cognitivebehavioural therapy as a favourable option because of their low costs compared with maintenance antidepressants. ${ }^{47,48}$ Future studies are needed to examine at what point in time a break-even point will appear where costs and outcomes will improve for PCT/-AD.

\section{Limitations}

Some limitations have to be acknowledged. First, we collected the cost and QALY data via online questionnaires that were not mandatory, resulting in missing data. We handled missing data using multiple imputations. Baseline variables predicted whether data were missing, suggesting data were at least partly missing at random and that multiple imputations may have reduced bias associated with complete case analyses. In addition, our sensitivity analyses suggested that missing data had no substantial influence on the results. However, a possibility remains that missing data were not missing at random. Second, this study was not powered to detect a significant difference in QALYs and costs. Yet, the probabilistic and medical decision-making techniques we used allowed us to make estimations of the cost-effectiveness and cost-utility. 
Third, whereas a willingness-to-pay threshold for QALYs is available, this is not the case for depression-free days. Therefore, interpretation of the willingness to pay regarding depression-free days remains speculative. Given that in the current study most outcomes were located in the south-east quadrant where costs were lower and effects better, this limitation does not have a major impact on the implications of this study. Fourth, no pill-placebo control group or control group for PCT was used, which would have enabled examination of the specific effects of antidepressants and PCT. Fifth, the data were collected in the Netherlands and the study comprised individuals in remission from at least two previous major depressive episodes using maintenance antidepressants, which might compromise generalisability. Sixth, the estimated budget impact relies on the assumption that the trial results are generalisable to a larger population within the Netherlands, which might not be the case. Nevertheless, we believe that our results can be generalised as participants were recruited via a wide range of resources and our inclusion and exclusion criteria regarding comorbidity were lenient.

\section{Clinical implications and recommendations}

For individuals willing to stay on antidepressants, the addition of PCT provides a substantial benefit over antidepressants only in terms of cost-effectiveness and the budget impact. For individuals wishing to taper antidepressants, extra investments might be required. Since preventive effects of psychological interventions last up to 10 years $^{4,18}$ and maintenance antidepressants generate long-term costs, future studies should examine the cost-effectiveness of relapse prevention programmes in maintenance antidepressants over a longer time span than the 24 months of the current study. In addition, future studies should examine how withdrawal symptoms and specific patterns of discontinuing antidepressants are associated with cost-effectiveness and whether PCT should be administered before or after tapering antidepressants.

Nicola S. Klein, PhD Candidate, Department of Clinical Psychology and Experimental Psychopathology, University of Groningen; and Psychologist, Top Referent Traumacentrum, GGZ Drenthe, the Netherlands; Ben F. M. Wijnen, Health Economist, Center of Economic Evaluation, Trimbos Institute (Netherlands Institute of Mental Health and Addiction); and Postdoctoral Researcher, Department of Health Services Research, Maastricht University, Care and Public Health Research Institute CAPHRI, the Netherlands; Joran Lokkerbol, Director, Center of Economic Evaluation, Trimbos Institute (Netherlands Institute of Mental Health and Addiction), the Netherlands; and Harkness Fellow in Health Care Policy and Practice, Department of Health Care Policy, Harvard Medical School, USA; Erik Buskens, Professor of Health Technology Assessment, Faculty of Economics and Business, University Medical Center Groningen, University of Groningen, the Netherlands; Hermien J. Elgersma, PhD Candidate, Department of Clinical Psychology and Experimental Psychopathology, University of Groningen; and Clinical Psychologist, Accare, the Netherlands; Gerard D. van Rijsbergen, Health Care Psychologist, Department of Early Detection and Intervention in Psychosis, GGZ Drenthe, the Netherlands; Christien Slofstra, Senior Researcher, Lentis Psychiatric Institute, Lentis Research, the Netherlands; Johan Ormel, Professor of Psychiatric Epidemiology, University Center for Psychiatry and Interdisciplinary Center Psychiatric Epidemiology, University of Groningen, University Medical Center Groningen, the Netherlands; Jack Dekker, Professor, Department of Clinical, Neuro and Developmental Psychology, Vrije Universiteit; and Head of Research Department, Arkin Mental Health Institute, the Netherlands; Peter J. de Jong, Professor of Experimental Psychopathology, Chair of Department of Clinical Psychology and Experimental Psychopathology, University of Groningen, the Netherlands; Willem A. Nolen, Emeritus Professor, Department of Psychiatry, University of Groningen, University Medical Center Groningen, the Netherlands; Aart H. Schene, Professor of Psychiatry, Head of the Department of Psychiatry, Radboud University Medical Center; and Principal Investigator, Donders Institute for Brain, Cognition and Behavior, Radboud University, the Netherlands; Steven D. Hollon, Professor of Psychology, Department of Psychology, Vanderbilt University, USA; Huibert Burger, Associate Professor of Clinical Epidemiology, Department of General Practice, University of Groningen, University Medical Center Groningen; and Associate Professor of Clinical Epidemiology, Amsterdam UMC, location AMC, Department of Psychiatry, University of Amsterdam, the Netherlands: Claudi L. H. Bockting, Professor of Clinical Psychology in Psychiatry, Amsterdam UMC, location AMC, Department of Psychiatry, University of Amsterdam, the Netherlands

Correspondence: Claudi L. H. Bockting, Department of Psychiatry, University of Amsterdam, Meibergdreef 9, 1105 AZ Amsterdam; University of Groningen, Grote Kruisstraat 2/19712 TS Groningen, the Netherlands. Email: c.l.bockting@amc.uva.n

First received 24 Jul 2018, final revision 31 Oct 2018, accepted 26 Nov 2018

\section{Funding}

The current study was sponsored by the Netherlands Organisation for Health Research and Development (ZONMW) (171002401). In addition, C.L.H.B. worked on this manuscript during a fellowship at the Netherlands Institute for Advanced study in the Humanities and Social Sciences, supported by the Royal Netherlands Academy of Arts and Sciences.

\section{Acknowledgements}

We would like to thank all participants for their investment in the study and all psychologists, general practitioners and psychiatrists providing the treatment. In addition, we thank all assessors and assistants for their contribution.

\section{Supplementary material}

Supplementary material is available online at https://doi.org/10.1192/bjo.2018.81.

\section{References}

1 Vos T, Abajobir AA, Abate KH, Abbafati C, Abbas KM, Abd-Allah F, et al. Global, regional, and national incidence, prevalence, and years lived with disability for 328 diseases and injuries for 195 countries, 1990-2016: a systematic analysis for the Global Burden of Disease Study 2016. Lancet 2017; 390: 1211-59.

2 Richards D. Prevalence and clinical course of depression: a review. Clin Psychol Rev 2011; 31: 1117-25.

3 Donohue JM, Pincus HA. Reducing the societal burden of depression: a review of economic costs, quality of care and effects of treatment. Pharmacoeconomics 2007; 25: 7-24.

4 Bockting CLH, Hollon SD, Jarrett RB, Kuyken W, Dobson K. A lifetime approach to major depressive disorder: the contributions of psychological interventions in preventing relapse and recurrence. Clin Psychol Rev 2015; 41: 16-26.

5 Borges S, Chen YF, Laughren TP, Temple R, Patel HD, David PA, et al. Review of maintenance trials for major depressive disorder: a 25-year perspective from the US Food and Drug Administration. J Clin Psychiatry 2014; 75: 205-14.

6 Geddes JR, Carney SM, Davies C, Furukawa TA, Kupfer DJ, Frank E, et al. Relapse prevention with antidepressant drug treatment in depressive disorders: a systematic review. Lancet 2003; 361: 653-61.

7 Glue P, Donovan MR, Kolluri S, Emir B. Meta-analysis of relapse prevention antidepressant trials in depressive disorders. Aust N Z J Psychiatry 2010; 44: 697-705.

8 Kaymaz N, Van Os J, Loonen AJ, Nolen WA. Evidence that patients with single versus recurrent depressive episodes are differentially sensitive to treatment discontinuation: a meta-analysis of placebo-controlled randomized trials. $J$ Clin Psychiatry 2008; 69: 1423-36.

9 Pampallona S, Bollini P, Tibaldi G, Kupelnick B, Munizza C. Patient adherence in the treatment of depression. Br J Psychiatry 2002; 180: 104-9.

10 McHugh K, Whitton S, Peckham A, Welge J, Otto M. Patient preference for psychological vs. pharmacological treatment of psychiatric disorders: a meta-analytic review. J Clin Psychol 2013; 74: 595-602.

11 Bockting CLH, Elgersma HJ, Van Rijsbergen GD, De Jonge P, Ormel J, Buskens E، et al. Disrupting the rhythm of depression: design and protocol of a randomized controlled trial on preventing relapse using brief cognitive therapy with or without antidepressants. BMC Psychiatry 2011; 11: 1-22.

12 Bockting CLH, Klein NS, Elgersma HJ, Van Rijsbergen GD, Slofstra C, Ormel J, et al. Effectiveness of preventive cognitive therapy while tapering antidepressants versus maintenance antidepressant treatment versus their combination in prevention of depressive relapse or recurrence (DRD study): a three-group, multicentre, randomised controlled trial. Lancet Psychiatry 2018; 5: 401-10.

13 Zorginstituut Nederland. Richtlijn voor het Uitvoeren van Economische Evaluaties in de Gezondheidszorg [Guideline for Conducting Economic Evaluations in Health Care.] Zorginstituut Nederland, 2015.

14 Husereau D, Drummond M, Petrou S, Carswell C, Moher D, Greenberg D, et al. Consolidated Health Economic Evaluation Reporting Standards (CHEERS) statement. Value Health 2013; 16: e1-5.

15 Spitzer RL, Williams JB, Gibbon M, First MB. The Structured Clinical Interview for DSM-III-R (SCID): I. History, rationale, and description. Arch Gen Psychiatry 1992; 49: 624-9.

16 Hamilton M. A rating scale for depression. J Neurol Neurosurg Psychiatry 1960; 23: 56-62.

17 Biesheuvel-Leliefeld KEM, Dijkstra-Kersten SMA, Van Schaik DJF, Van Marwijk HWJ, Smit F, Van der Horst HE, et al. Effectiveness of supported selfhelp in recurrent depression: a randomized controlled trial in primary care. Psychother Psychosom 2017; 86: 220-30. 
18 Bockting $\mathrm{CLH}$, Smid $\mathrm{NH}$, Koeter MW, Spinhoven P, Beck AT, Schene AH. Enduring effects of preventive cognitive therapy in adults remitted from recurrent depression: a 10 year follow-up of a randomized controlled trial. J Affect Disord 2015; 185: 188-94.

19 Bockting CLH, Schene AH, Spinhoven P, Koeter MW, Wouters LF, Huyser J, et al. Preventing relapse/recurrence in recurrent depression with cognitive therapy: a randomized controlled trial. J Consult Clin Psychol 2005; 73: 647-57.

20 Bockting CLH, Spinhoven P, Wouters LF, Koeter MW, Schene AH, DELTA Study Group. Long-term effects of preventive cognitive therapy in recurrent depression: a 5.5-year follow-up study. J Clin Psychiatry 2009; 70: 1621-8.

21 De jonge M, Bockting CLH, Van Dijk M, Van Schaik DJH, Peen J, Kikkert MJ, et al Preventing depressive relapse and recurrence in cognitive therapy responders: a multicenter randomized controlled trial: preventive cognitive therapy vs treatment as usual. Under review 2018.

22 Hakkaart-van Roijen L. Manual Trimbos/iMTA Questionnaire for costs Associated with Psychiatric IIIness (TiC-P). Institute for Medical Technology Assessment, 2002.

23 Bouwmans $C$, De Jong $\mathrm{K}$, Timman R, Zijlstra-Vlasveld $M$, Van der FeltzCornelis C, Tan Swan S, et al. Feasibility, reliability and validity of a questionnaire on healthcare consumption and productivity loss in patients with a psychiatric disorder (TiC-P). BMC Health Serv Res 2013; 13: 217

24 Koopmanschap MA, Rutten FF, Van Ineveld BM, Van Roijen L. The friction cost method for measuring indirect costs of disease. J Health Econ 1995; 14 171-89.

25 Zorginstituut Nederland. Kostenhandleiding: Methodologie van Kostenonderzoek en Referentieprijzen voor Economische Evaluaties in de Gezondheids zorg. Geactualiseerde Versie 2015 [Cost Guide: Methodology of Cost Research and Reference Prices for Economic Evaluations in Health Care. Updated version 2015.] Zorginstituut Nederland, 2015.

26 Rabin R, De Charro F. EQ-5D: a measure of health status from the EuroQol Group. Ann Med 2001; 33: 337-43.

27 Lamers LM, McDonnell J, Stalmeier PF, Krabbe PF, Busschbach JJ. The Dutch tariff: results and arguments for an effective design for national EQ-5D valuation studies. Health Econ 2006; 15: 1121-32.

28 White IR, Carlin JB. Bias and efficiency of multiple imputation compared with complete-case analysis for missing covariate values. Stat Med 2010; 29 : 2920-31.

29 Manca A, Palmer S. Handling missing data in patient-level cost-effectiveness analysis alongside randomised clinical trials. Appl Health Econ Policy 2005; 4 65-75.

30 Drummond MF, Sculpher MJ, Torrance GW, O'Brien BJ, Stoddart GL. Methods for the Economic Evaluation of Health Care Programmes (3rd edn). Oxford University Press, 2005

31 Efron B, Tibshirani RJ. An Introduction to the Bootstrap. Chapman \& Hall/CRC, 1994.

32 Black WC. The CE plane: a graphic representation of cost-effectiveness. Med Decis Making 1990; 10: 212-4.

33 Fenwick E, O'Brien BJ, Briggs A. Cost-effectiveness acceptability curves - facts fallacies and frequently asked questions. Health Econ 2004; 13: 405-15.

34 Lokkerbol J, Adema D, Cuijpers P, Reynolds CF, Schulz R, et al. Improving the cost-effectiveness of a healthcare system for depressive disorders by implementing telemedicine: a health economic modeling study. Am J Geriatr Psychiatry 2014; 22: 253-62.

35 Brazier J, Connell J, Papaioannou D, Mukuria C, Mulhern B, Peasgood T, et al. A systematic review, psychometric analysis and qualitative assessment of generic preference-based measures of health in mental health populations and the estimation of mapping functions from widely used specific measures. Health Technol Assess 2014; 18: 1-188.

36 Huijbers MJ, Spinhoven P, Spijker J, Ruhé HG, Van Schaik DJ, Van Oppen P, et al. Adding mindfulness-based cognitive therapy to maintenance antidepressant medication for prevention of relapse/recurrence in major depressive disorder: randomised controlled trial. J Affect Disord 2015; 187: 54-61.

37 Guidi J, Tomba E, Fava GA. The sequential integration of pharmacotherapy and psychotherapy in the treatment of major depressive disorder: a meta-analysis of the sequential model and a critical review of the literature. Am J Psychiatry 2016; 173: 128-37.

38 Scott J, Palmer S, Paykel E, Teasdale J, Hayhurst H. Use of cognitive therapy for relapse prevention in chronic depression. Cost-effectiveness study. $\mathrm{Br} J$ Psychiatry 2003; 182: 221-7.

39 Shimodera S, Furukawa TA, Mino Y, Shimazu K, Nishida A, Inoue S. Cost-effectiveness of family psychoeducation to prevent relapse in major depression: results from a randomized controlled trial. BMC Psychiatry 2012; 12: 1-6.

40 Karyotaki E, Tordrup D, Buntrock C, Bertollini R, Cuijpers P. Economic evidence for the clinical management of major depressive disorder: a systematic review and quality appraisal of economic evaluations alongside randomised controlled trials. Epidemiol Psychiatr Sci 2017; 26: 501-16.

41 Kuyken W, Byford S, Taylor RS, Watkins E, Holden E, White K, et al. Mindfulnessbased cognitive therapy to prevent relapse in recurrent depression. J Consult Clin Psychol 2008; 76: 966-78.

42 Kuyken W, Hayes R, Barrett B, Byng R, Dalgleish T, Kessler D, et al. Effectiveness and cost-effectiveness of mindfulness-based cognitive therapy compared with maintenance antidepressant treatment in the prevention of depressive relapse or recurrence (PREVENT): a randomised controlled trial. Lancet 2015; 386: 63-73.

43 Fava GA, Gatti A, Belaise C, Guidi J, Offidani E. Withdrawal symptoms after selective serotonin reuptake inhibitor discontinuation: a systematic review. Psychother Psychosom 2015; 84: 72-81.

44 Gauthier G, Guérin A, Zhdanava M, Jacobson W, Nomikos G, Merikle E, et al. Treatment patterns, healthcare resource utilization, and costs following firstline antidepressant treatment in major depressive disorder: a retrospective US claims database analysis. BMC Psychiatry 2017; 17: 1-12.

45 Katz G. Tachyphylaxis/tolerance to antidepressive medications: a review. Isr J Psychiatry Relat Sci 2011; 48: 129-35.

46 Andrews PW, Kornstein SG, Halberstadt LJ, Gardner CO, Neale MC. Blue again: perturbational effects of antidepressants suggest monoaminergic homeostasis in major depression. Front Psychol 2011; 159: 1-24.

47 Prukkanone B, Vos T, Bertram M, Lim S. Cost-effectiveness analysis for antidepressants and cognitive behavioral therapy for major depression in Thailand. Value Heal 2012; 15 (suppl 1): S3-8

48 Vos T, Corry J, Haby MM, Carter R, Andrews G. Cost-effectiveness of cognitive behavioural therapy and drug interventions for major depression. Aust $\mathrm{N} Z \mathrm{~J}$ Psychiatry 2005: 39: 683-92. 